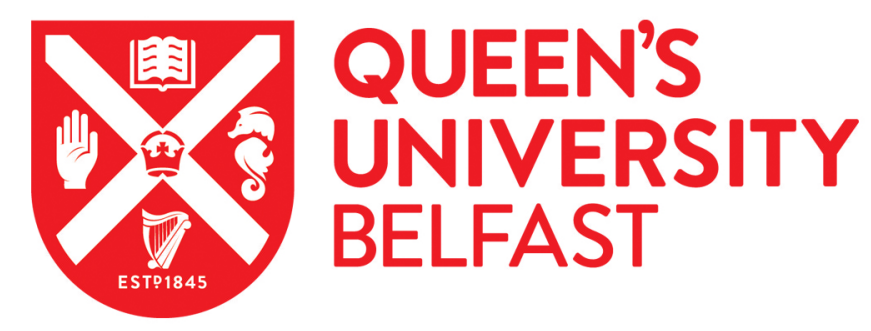

\title{
Multifunctional and robust composite materials comprising gold nanoparticles at a spherical polystyrene particle surface
}

Belhout, S. A., Kim, J. Y., Hinds, D. T., Owen, N. J., Coulter, J. A., \& Quinn, S. J. (2016). Multifunctional and robust composite materials comprising gold nanoparticles at a spherical polystyrene particle surface. Chemical Communications, 52(100), 14388-14391. https://doi.org/10.1039/c6cc07947d

Published in:

Chemical Communications

Document Version:

Peer reviewed version

Queen's University Belfast - Research Portal:

Link to publication record in Queen's University Belfast Research Portal

Publisher rights

(C) 2017 Royal Society of Chemistry.

This work is made available online in accordance with the publisher's policies.

\section{General rights}

Copyright for the publications made accessible via the Queen's University Belfast Research Portal is retained by the author(s) and / or other copyright owners and it is a condition of accessing these publications that users recognise and abide by the legal requirements associated with these rights.

Take down policy

The Research Portal is Queen's institutional repository that provides access to Queen's research output. Every effort has been made to ensure that content in the Research Portal does not infringe any person's rights, or applicable UK laws. If you discover content in the Research Portal that you believe breaches copyright or violates any law, please contact openaccess@qub.ac.uk. 


\section{Multifunctional and robust composite materials comprising gold nanoparticles at a spherical polystyrene particle surface}

Received 00th January 20xx, Accepted 00th January 20xx

\author{
Samir A. Belhout, ${ }^{a}$ Ji-Yoon Kim, ${ }^{a}$ David T. Hinds, ${ }^{a}$ Natalie J. Owen, ${ }^{b}$ Jonathan A. Coulter ${ }^{b}$ and Susan \\ J. Quinn ${ }^{\text {a* }}$
}

The preparation of a family of composite particles comprising gold nanoparticles (AuNP) assembled at a polystyrene (PS) surface is reported. Tunable loading is demonstrated for AuNP sizes (4.5-26 $\mathrm{nm})$. The robust composites are stable to multiple centrifugation and redispersion cycles and to conditions of high ionic strength, physiological buffer and cell culture media. These properties provide potential for a variety of applications from cellular studies to catalysis.

Access to gold metal nanoparticles (AuNPs) of defined size ${ }^{1}$ and shape, including rods, ${ }^{2 \mathrm{a}}$ prisms, $^{2 \mathrm{~b}}$ stars $^{2 \mathrm{c}}$ and cubes, ${ }^{2 \mathrm{~d}}$ is increasingly possible through a variety of preparative methods. These AuNPs display unique optical, mechanical, electrical and chemical properties, ${ }^{3}$ which have been widely applied in catalysis, ${ }^{4}$ surface enhanced Raman spectroscopy (SERS), ${ }^{5}$ and nanomedicine. ${ }^{6}$ In the area of nanomedicine they have been employed in light activated release of therapeutic payloads by NP heating, ${ }^{7}$ and AuNP radiosensitization, which is a novel approach to enhance the ionising radiation dose in radiotherapy. ${ }^{8}$ Harnessing the intrinsic properties of metal NPs typically requires them to be stable to aggregation. In addition, the sensitivity of the surface plasmon resonance (SPR) band to the relative location of particles and their interparticle distance ${ }^{9}$ makes the controlled organisation and localisation of AuNPs attractive as an avenue to plasmonic structures with customised optical properties. ${ }^{9 c}$ One approach that can potentially satisfy these requirements is the preparation of supported AuNPs composite particles. ${ }^{10}$ These have the advantage of being compatible with media, including biological media, that typically lead to NP agglomeration, are more robust to recycling and easier to handle and process. Numerous methodologies have been used to prepare supported AuNPs. These include the direct immobilisation of preformed AuNP on the support, ${ }^{10}$ and seeded $^{11}$ or unseeded ${ }^{12}$ in-situ AuNP synthesis. The direct immobilisation of well-defined AuNPs typically exploits the affinity of gold for amine, thiol and phosphine functional groups present at the support surface. A variety of supports have been reported including PS beads, which have yielded composite

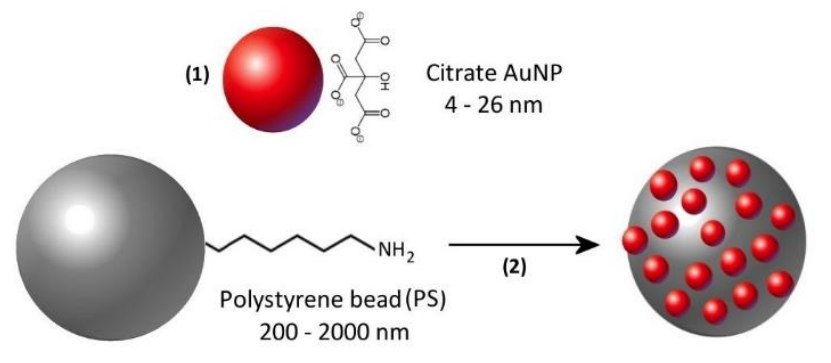

AuNP@PS composite

Scheme 1 Schematic representation of two step composite formation of AuNP@PS

particles with varying coverage of AuNPs. ${ }^{13}$ Yet control of the population of AuNPs on the surface of the composites, and the impact on the resulting optical properties, remains challenging. In this study we report the controlled assembly of AuNPs on amine modified PS beads, their comprehensive characterisation, resulting optical properties and their stability to biological media. The versatile method is applied to a range of AuNP and PS support sizes and leads to the formation of robust colloidal materials with tunable and high loadings. In particular, we investigate the stability of the composites to media that would typically lead to aggregation. We demonstrate the potential applicability of the composites in cellular uptake and catalysis.

PS supported AuNP composite particles were prepared using a two-step procedure (Scheme 1). In step one, citrate stabilised gold NPs of different sizes (see Fig. S1) were prepared; $4.5 \mathrm{~nm}$ NPs were synthesised in high concentration by modifying the nm) NPs were obtained using a seed growth method. ${ }^{1 \mathrm{c}}$ The concentration of AuNPs was determined using the NP radius, as per the method reported by Jana et. al (see SI), ${ }^{14}$ while larger $(15$ to 50 determined by TEM, the ppm of Au in solution from atomic

\footnotetext{
a. School of Chemistry, University College Dublin, Belfield, Dublin 4, Ireland

b. School of Pharmacy, Queen's University Belfast, Belfast, BT9 7BL, UK

Electronic Supplementary Information (ESI) available: For details of methods used and characterization of AuNPs and composite particles including TEM, UV-vis, DLS and zeta potential see DOI: 10.1039/c5cc01543j See DOI: 10.1039/x0xx00000x
} 
absorption spectroscopy (AAS) and the density of bulk gold (see Table S1). In the second step, AuNPs were self-assembled onto the PS bead under mild shaking (1000 rpm for $1 \mathrm{~h}$, see SI). The affinity of the PS surface amine groups for gold resulted in the efficient binding, which was evident from a loss of colour from the AuNP solution. The excess AuNPs were removed by centrifugation washing and the composites are found to be stable to repeated cycles of centrifugation-dispersion without loss of the surface AuNPs (see Fig. S2). The as formed AuNP@PS were fully characterised by UV-visible spectroscopy, dynamic light scattering (DLS), $\xi$-potential measurements and SEM.

The loading of $15 \mathrm{~nm}$ AuNPs at the surface of $200 \mathrm{~nm}$ PS was investigated by varying the concentration of AuNPs in the presence of a fixed amount of PS. The supernatant, obtained after washing by centrifugation, was analysed by measuring (a) the absorption of the SPR band and (b) the ppm of Au by AAS, see Fig 1a. At stoichiometric ratios greater than 120 AuNPs to 200 nm PS, free 'unbound' AuNPs were detected in the supernatant, which indicated that the surface was fully loaded. The maximum loading capacity was determined from the intercept of the AuNPs concentrations pre and post the saturation point. The results obtained from UV-vis and AAS measurements were found to be in close agreement, $124 \pm 5$ AuNPs and $138 \pm 3$ AuNPs per PS sphere, respectively. In addition, AAS analysis of fully occupied composite after washing yielded a value of maximum loading of $131 \pm 11$ AuNPs per PS, which is in excellent agreement with the values obtained from the supernatant measurements.

Using the data in Fig 1a. three different loading domains were identified, namely; low $0-40 \%$ (I), moderate $41-80 \%$ (II) and high $81-100 \%$ (III), see Fig 1b. Access to tunable loadings corresponding to these domains was confirmed by SEM (Fig 1c). The SEM images also reveal an absence of free gold, which is successfully removed under the mild washing conditions. It

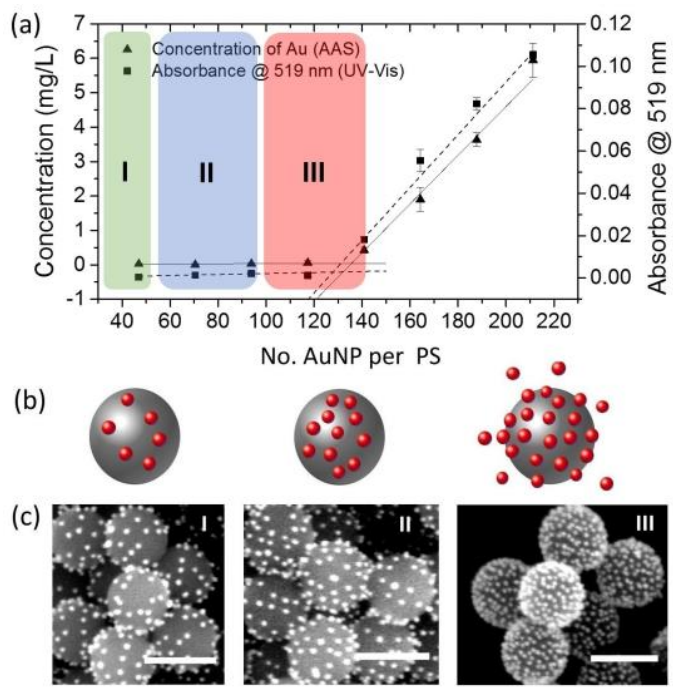

Figure 1. $(a, b)$ UV-vis and AAS determination of the maximum loading of $15 \mathrm{~nm}$ AuNPs on $200 \mathrm{~nm}$ PS with low (I), medium (II) and maximum loading (III). (c) Representative SEM images (scale bar $=100 \mathrm{~nm}$ ).
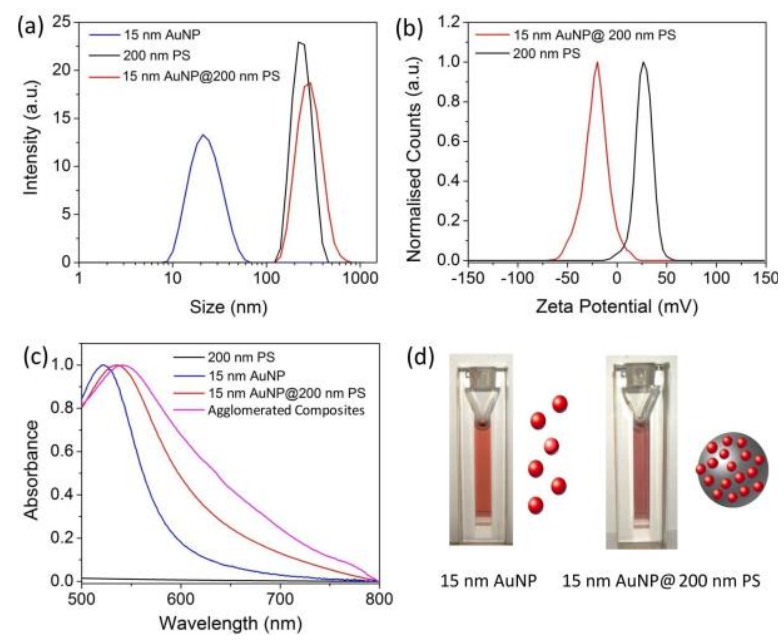

Figure 2. (a) DLS (b) Zeta potential and (c) UV-vis measurements of AuNPs, 'bare' PS and 15 nm AuNP@200 nm PS in water. (d) Image showing the solution of AuNPs and 15 nm AuNP@200 nm PS in water

should be noted that PS beads with AuNP loadings significantly below the maximum occupancy (in the region of $<70 \%$, see Fig. S3) were found to agglomerate due to AuNP binding to more than one PS bead. This eventually resulted in the particles falling out of solution though is temporarily reversible under sonication.

The formation of fully loaded 15 nm AuNP@200 nm PS resulted in an increase in hydrodynamic size by approximately $50 \mathrm{~nm}$, see Fig. 2a (Table S2). Importantly, the discrete nature of the composite particles was confirmed by the absence of a DLS signal associated with higher order aggregated systems. The immobilisation of the AuNPs yielded a change in the $\xi$-potential from $+27 \mathrm{mV}$ to $-19 \mathrm{mV}$ (pH 7.4). This is expected due to binding of the citrate stabilised (negatively charged) AuNPs.

The visible absorption spectra recorded for the $15 \mathrm{~nm}$ AuNP@200 nm PS revealed a shift in the SPR band of the AuNPs from $519 \mathrm{~nm}$ to $535 \mathrm{~nm}$, see Fig. 2c-d. This change is attributed to the interaction of the AuNPs, with (i) the PS surface and (ii) adjacent surface bound AuNPs. ${ }^{15}$ The relative contributions of these interactions were assessed by considering the SPR position for sparsely loaded PS, which was found to shift by $2 \mathrm{~nm}$ (see Fig. S3). This suggests that the AuNPs are not significantly embedded in the PS, whose greater refractive index would be expected to significantly influence the SPR position. The agglomeration of composite particles resulted in a further red-shift in the SPR band position and broadening of the band, see Fig. 2c. Subtraction of the spectrum obtained for the discrete $15 \mathrm{~nm}$ AuNP@200 nm PS from that obtained for the agglomerated composite reveals a loss in the signal at $535 \mathrm{~nm}$ and the appearance of a contribution at $\sim 600 \mathrm{~nm}$ which is characteristic of aggregated AuNPs (see Fig. S4).

The assembly of $4.5 \mathrm{~nm}$ and $26 \mathrm{~nm}$ AuNPs at the surface of 200 nm PS beads (Fig. S5-S8) was also demonstrated. Analysis of the supernatant was again used to determine the maximum loading capacity, which was found to be $\sim 980$ for $4.5 \mathrm{~nm}$ and 
Table 1 Summary of composite loadings

\begin{tabular}{cccc}
\hline $\begin{array}{c}\text { Composite } \\
\text { AuNP@PS Bead }\end{array}$ & a Maximum Loading AuNP & Maximum Loading \\
UVis & AAS & Composite AAS \\
\hline $4.5 \mathrm{~nm} @ 200 \mathrm{~nm}$ & $977 \pm 6$ & $1015 \pm 11$ & $978 \pm 48$ \\
$15 \mathrm{~nm} @ 200 \mathrm{~nm}$ & $124 \pm 5$ & $138 \pm 3$ & $131 \pm 11$ \\
$26 \mathrm{~nm} @ 200 \mathrm{~nm}$ & $30 \pm 2$ & $28 \pm 1$ & $32 \pm 3$ \\
$15 \mathrm{~nm} @ 2000 \mathrm{~nm}$ & $7429 \pm 273$ & $7211 \pm 183$ & $7622 \pm 453$
\end{tabular}

${ }^{\mathrm{a}}$ Determined from analysis of the supernatant.

$\sim 30$ for $26 \mathrm{~nm}$ AuNPs. Again the results reveal a good agreement for the loading determined by UV-visible and AAS analysis of the supernatant and the AAS values obtained from the acid digested composites (Table 1). TEM images of the different assemblies are shown in Fig. 3, importantly all three systems show the absence of any free or aggregated AuNPs.

The description of metal NP loading is typically related to the particular application of interest and can be given in terms of (a) the number of NPs, (b) the total NP surface area and (c) the \%wgt of NPs, all per composite weight (see Table S5). The preparation of the composite families described herein gives access to stable, dispersible (Fig. S9) systems with a broad range of AuNP loadings from 6 to $55 \% \mathrm{wgt}$, which are suitable for different applications. One feature of all the composites is the observation of a high percentage of discrete surface AuNPs. This suggests that the assembly at the surface is directed by electrostatic repulsion between the negatively charged AuNPs. Such an electrostatic repulsion driven assembly has been observed for the self-assembly of AuNPs coated in a - $\mathrm{COOH}$ containing polymer. $^{16}$

A key property of NP composite materials is their increased stability to environments that lead to irreversible aggregation of the free AuNPs. For application in sensing and nanomedicine, it is necessary to consider the stability to the solution environment. The time dependent stability of the composites to conditions of ionic strength (100 to $500 \mathrm{mM} \mathrm{NaCl}$ ) was monitored by UVvisible spectroscopy. The $4.5 \mathrm{~nm}$ and $15 \mathrm{~nm}$ composites were found to be stable up to $350 \mathrm{mM} \mathrm{NaCl}$, which is almost twice the highest physiological $\mathrm{NaCl}$ concentration, ${ }^{17}$ with no evidence for aggregation observed over $24 \mathrm{~h}$ and $80 \%$ retained at $500 \mathrm{mM}$,
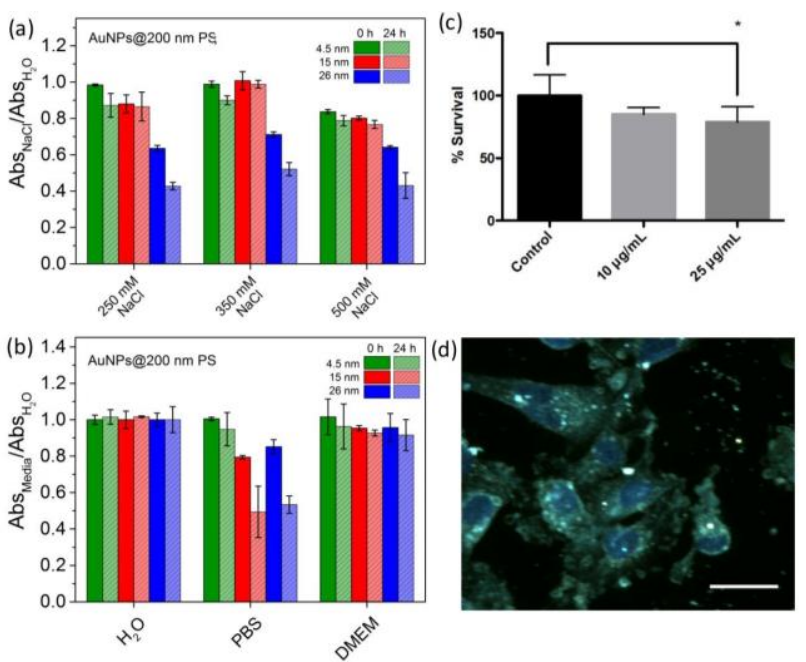

Figure 4 Stability of composites to (a) ionic strength and (b) biologically relevant environments.4.5 nm AuNP@200 nm PS cellular study: (c) Alamar Blue cytoxicity results and (d) Fluorescent dark field image of uptake by MDA-MB-231 cells.

see Fig. 4a and Fig. S11-S13. Though some loss of sample was observed in the case of the $26 \mathrm{~nm}$ AuNP@PS. In contrast, the free AuNPs were found to irreversibly aggregate in $100 \mathrm{mM}$ $\mathrm{NaCl}$, see Fig. S14. The $4.5 \mathrm{~nm}$ AuNP@PS also exhibited excellent stability in phosphate buffered saline (PBS), pH 7.4, with over $90 \%$ of the sample retained, see Fig. 4b. While all composites exhibited excellent stability in cell culture medium (DMEM, Dulbecco's Modified Eagle Medium, supplemented with $10 \%$ Foetal Bovine Serum, FBS) over a 72 h period, Fig. S16, with $<10 \%$ loss over a $6 \mathrm{~h}$ incubation period, Fig. S17.

Due to their high NP loading and stability the composites are potential radiosensitisaton agents. Given the excellent stability demonstrated by the $4.5 \mathrm{~nm}$ composites, their cellular uptake was investigated. Human breast adenocarcinoma cells (MDA-MB231) were chosen as they are a potential target for future studies. The short-term cytoxicity, assessed using the Alamar Blue assay,
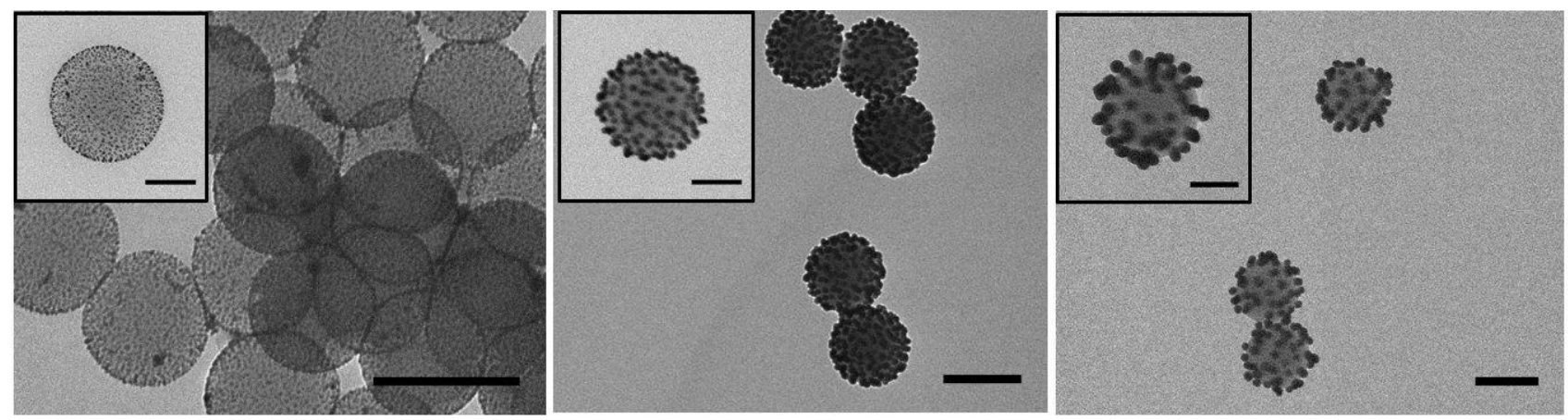

Figure 3 Transmission electron microscopy characterisation of (left) 4.5 nm AuNP@200 nm PS composite; (centre) 15 nm AuNP@200 nm PS composite and (right) $26 \mathrm{~nm}$ AuNP@200 nm composite. Main scale bar $200 \mathrm{~nm}$ and inset scale bar $100 \mathrm{~nm}$. 
showed low toxicity for the composites at 10 and $25 \mu \mathrm{g} \mathrm{mL}^{-1}$, see Fig. 4c. The uptake of the composites after $6 \mathrm{~h}$ incubation was confirmed by fluorescent dark field imaging, see Fig. 4d. This showed significant cytoplasmic scattering, with evidence of strong perinuclear accumulation, pointing towards clatherindependent endocytosis as previously reported. ${ }^{18}$ In contrast, the untreated cells appear comparatively dark, with only high density, protein rich cytoplasmic regions generating a low intensity signal, Fig. S18. The morphological similarity between control and treated cells supports the preliminary evidence of biocompatibility provided by the Alamar Blue assay.

The multifunctional applicability of the composite particles was further explored by considering their catalytic behaviour. For this purpose, larger, $15 \mathrm{~nm}$ AuNPs @2000 nm PS composites were prepared, see Fig. S19-20, which demonstrated the versatility of the approach. The loading capacity was found to be $\sim 7500$ AuNPs per PS bead. While these larger composites were found to readily sediment with time, they were easily redispersed by shaking or mild sonication. SEM images confirmed the formation of the composites and also revealed a majority of discrete AuNPs at the surface, which is reflected in the UVvisible spectrum recorded for the composite (Fig. S20). The catalytic activity was tested using the model reaction of the reduction of para-nitrophenol in the presence of an excess amount of sodium borohydride $\left(\mathrm{NaBH}_{4}\right)$, see Fig. S21. ${ }^{4 a}$ Similar to previous observations a delay time was observed after which the reaction proceeded within $200 \mathrm{~s}$ with a half-life of $46 \mathrm{~s}$. Future work will consider the role of AuNP separation on the reaction kinetics observed for this model reaction. ${ }^{4} \mathrm{e}$

In summary, we have demonstrated the assembly of a family of robust AuNPs composite particles whose tunable NP loading has been determined using complementary techniques of UVvisible and AA spectroscopy and confirmed by electron microscopy. The stability of the composites was demonstrated in a variety of media. In particular, the composites are found to be stable to varying ionic strength and physiological buffer for long periods of time. Preliminary cell studies revealed uptake of the $4.5 \mathrm{~nm} @ 200 \mathrm{~nm}$ PS which is well tolerated by the cells, with low associated toxicity. In addition, the catalytic activity for the reduction was demonstrated, which confirms the accessibility of the immobilised AuNPs to solution molecules. The robust and multifunctional composites are expected to find use in a variety of additional applications. One example of this is in SERS, which can exploit (i) the loading dependent agglomeration and (ii) surface AuNP interactions. ${ }^{5 a}$ Furthermore, as the AuNP optical properties are modulated upon assembly the formation of plasmonic composites can be used to access tunable optical properties (see Table S6). In particular, their low toxicity and cellular uptake opens the door to potential applications in nanomedicine including radiosensitisation.

This work was supported by the Irish Research Council (GOIP/2013/1110, SAB) and UCD. We are grateful to Prof. Kenneth Dawson for access to the DLS instrumentation and to
Dr. Heath Bagshaw at the CMA in TCD for assistance with electron microscopy measurements.

\section{Notes and references}

1 (a) J. Turkevich, P. C. Stevenson and J. Hillier, Discuss. Faraday Soc., 1951, 11, 55-75. (b) M. Brust, M. Walker, D.Bethell, D. J. Schiffrin and R. Whyman, Chem. Commun., 1994, 801-802. (c) N. G. Bastús, J. Comenge and V. Puntes, Langmuir, 2011, 27, 11098-11105.

2 (a) A. Gole and C. J. Murphy, Chem. Mater., 2004, 16, 3633 3640. (b) S. S. Shankar, A. Rai, B. Ankamwar, A. Singh, A. Ahmad and M. Sastry, Nat. Mat. 2004, 3, 482-488. (c) P.S. Kumar et al. Nanotechnology, 2008, 19, 015606. (d) H.-L. Wu, H.-R. Tsai, Y.-T. Hung, K.-U. Lao, C.-W. Liao, P.-J. Chung, J.-S. Huang, I-C. Chen, and M. H. Huang Inorg. Chem., 2011, 50, 8106-8111.

3 (a) M.-C. Daniel and D. Astruc, Chem. Rev., 2004, 104, $293-$ 346. (b) S. Eustis and M. a el-Sayed, Chem. Soc. Rev., 2006, 35, 209-217.

4 (a) S. Panigrahi, S. Basu, S. Praharaj, S. Pande, S. Jana, A. Pal, S. K. Ghosh and T. Pal, J. Phys. Chem. C, 2007, 111, 4596-4605. (b) M. Stratakis and H. Garcia, Chem. Rev. 2012, 112, 4469-4506. (c) J. M. Campelo, D. Luna, R. Luque, J. M. Marinas and A. A. Romero, ChemSusChem, 2009. 2, 1845. (d) C.-J. Jia and F. Schüth, Phys. Chem. Chem. Phys., 2011, 13, 2457-2487. (e) A. G. M. da Silva, T. S. Rodrigues, J. Wang, L. K. Yamada, T. V. Alves, F. R. Ornellas, R. A. Ando and P. H. C. Camargo, Langmuir, 2015, 31, 1027210278

5 (a) A. B. Serrano-Montes, J. Langer, M. Henriksen-Lacey, D. Jimenez de Aberasturi, D. M. Solís, J. M. Taboada, F. Obelleiro, K. Sentosun, S. Bals, A. Bekdemir, F. Stellacci and L. M. Liz-Marzán, J. Phys. Chem. C, 2016, 37, 20860-20868. (b) I. W. Guo, I. C. Pekcevik, M. C. P. Wang, B. K. Pilapil and B. D. Gates, Chem. Commun., 2014, 8157-8160.

6 E. Boisselier and D. Astruc, Chem. Soc. Rev., 2009, 38, 1759-1782.

7 A. Cifuentes-Rius, H. De Puig, J. C. Y. Kah, S. Borros and K. Hamad-Schifferli, ACS Nano, 2013, 7, 10066-10074. (b) Z. Xiao, C. Ji, J. Shi, E. M. Pridgen, J. Frieder, J. Wu and O. C. Farokhzad, Angew. Chemie Int. Ed., 2012, 51, $11853-11857$.

8 (a) C. Sicard-Roselli, E. Brun, M. Gilles, G. Baldacchino, C. Kelsey, H. McQuaid, C. Polin, N. Wardlow and F. J. Currell, Small, 2014, 10, 3338-3346. (b) S. Jain, J. A. Coulter, A. R. Hounsell, K. T. Butterworth, S. J. McMahon, W. B. Hyland, M .F. Muir, G. R. Dickson, K. M.Prise, F. J. Currell, J. M. O'Sullivan and D. G. Hirst, Int J Radiat Oncol Biol Phys., 2011, 79, 531-539.

9 (a) P. K. Jain, W. Huang and M. A. El-Sayed, Nano Lett., 2007, 7, 2080-2088. (b) B. M. Reinhard, M. Sui, H. Agarwal, A. P. Alivasatos and J. Liphardt, Nano Lett., 2005, 5, 22462252. (c) A. I. Dolinnyi, J. Phys Chem C, 2015, 119, 49905001. (d) J. N. Anker, W. P.Hall, O. Lyandres, N. C. Shah, J. Zhao and R.P. Van Duyne, Nat. Mat., 2008, 7, 442-453.

10 (a) T. Ishida, K. Kuroda, N. Kinoshita, W. Minagawa and M. Haruta, J. Colloid Interface Sci., 2008, 323, 105-111. (b) M. Li and G. Chen, Nanoscale, 2013, 5, 11919-11927.

11 W. Wang, W. Cai, Y. Yang, H. Li, M. Cong and T. Chen, Mater. Chem. Phys., 2013, 142, 756-762.

12 (a) J. Zhou, F. Ren, W. Wu, S. Zhang, X. Xiao, J. Xu and C. Jiang, J. Colloid Interface Sci., 2012, 387, 47-55. (b) M. Kanahara, M. Shimomura and H.Yabu, Soft Matter, 2014, 10, 
275-280. (c) K.-T. Yong, Y. Sahoo, M. T. Swihart and P. N. Prasad, Colloids Surf., A, 2006, 290, 89-105.

13 (a) T. Taniguchi, T. Inada, T. Kashiwakura, F. Murakami, M. Kohri and T. Nakahira Colloids Surf., A, 2011, 377, 6369. (b) M. Li and G. Chen, Nanoscale, 2013, 5, 1191911927. (c) H. Bao, T. Bihr, A.-S. Smith and R. N. K. Taylor Nanoscale, 2014, 6, 3954-3966.

14 N. Jana, L. Gearheart and C. Murphy, Langmuir, 2001, 17, 6782-6786.

15 S. Borsley, S. Flook and E. R. Kay, Chem. Commun., 2015, $7812-7815$.

16 W. Li, I. Kanyo, C.-H. Kuo, S. Thanneeru and J. He, Nanoscale, 2015, 7, 956-964.

17 C. A. Terry, M. J. Fernández, L. Gude L, A. Lorente and K. B. S. Grantt, Biochemistry, 2011, 50,10375-10389.

18 J. A. Coulter, S. Jain, K. T. Butterworth, L. E. Taggart, G. R. Dickson, S. J. McMahon, W. B. Hyland, M. F. Muir, C.

Trainor, A. R. Hounsell, J. M. O'Sullivan, G. Schettino, F. J. Currell, D. G. Hirst and K. M. Prise. Int. J. Nanomed., 2012, 7, 2673-2685. 